\title{
*Validation of the Assessment of Depression Inventory (ADI) Feigning Scale and clinical, demographic, and criminal profile differences between probable malingerers and psychiatric inpatients
}

Julia Marie Messer

West Virginia University

Follow this and additional works at: https://researchrepository.wvu.edu/etd

\footnotetext{
Recommended Citation

Messer, Julia Marie, "*Validation of the Assessment of Depression Inventory (ADI) Feigning Scale and clinical, demographic, and criminal profile differences between probable malingerers and psychiatric inpatients" (2008). Graduate Theses, Dissertations, and Problem Reports. 4405.

https://researchrepository.wvu.edu/etd/4405

This Dissertation is protected by copyright and/or related rights. It has been brought to you by the The Research Repository @ WVU with permission from the rights-holder(s). You are free to use this Dissertation in any way that is permitted by the copyright and related rights legislation that applies to your use. For other uses you must obtain permission from the rights-holder(s) directly, unless additional rights are indicated by a Creative Commons license in the record and/ or on the work itself. This Dissertation has been accepted for inclusion in WVU Graduate Theses, Dissertations, and Problem Reports collection by an authorized administrator of The Research Repository @ WVU. For more information, please contact researchrepository@mail.wvu.edu.
} 
Validation of the Assessment of Depression Inventory (ADI) Feigning Scale and Clinical, Demographic, and Criminal Profile Differences between Probable Malingerers and Psychiatric Inpatients

\author{
Julia Marie Messer
}

\author{
Dissertation submitted to the \\ Eberly College of Arts and Sciences \\ at West Virginia University \\ in partial fulfillment of the requirements \\ for the degree of
}
Doctor of Philosophy
in
Psychology
William Fremouw, Ph.D., Chair
Stanley Cohen, Ph.D.
Kevin Larkin, Ph.D.
Edward Baker, Ph.D.
Neil Mogge, Ph.D.

\title{
Department of Psychology
}

\author{
Morgantown, West Virginia \\ 2008
}

Keywords: Malingering, Assessment, Depression, Profile Differences 


\begin{abstract}
Validation of the Assessment of Depression Inventory (ADI) Feigning Scale and Clinical, Demographic, and Criminal Profile Differences between Probable Malingerers and Psychiatric Inpatients
\end{abstract}

Julia Marie Messer

Using archival data, "gold standard" assessment measures were used to determine group placement of psychiatric inpatients. One of two multi-scale measures of personality, the Minnesota Multiphasic Personality Inventory II (MMPI-2) or the Personality Assessment Inventory (PAI), were administered, as well as one of two interview measures designed specifically to assess for malingering, the Structured Interview of Reported Symptoms (SIRS) and/or the Miller Forensic Assessment of Symptoms Test (M-FAST). Based on either the MMPI-2 or the PAI and the SIRS, subjects were placed into one of three groups: nonmalingering inpatients, probable malingerers, and indeterminate malingerers. One purpose of identifying these groups was to validate the Assessment of Depression Inventory (ADI) Feigning scale $(\mathrm{Fg})$ with a known group of malingering inpatients who met dual criteria standards. Using the two most extreme groups, the non-malingering inpatients and the probable malingerers, the Fg scale cut score of 14 was highly effective in differentiating honest presentations from those likely feigned, with an $87.7 \%$ hit rate in correct identification of group membership. In addition, a chart review was conducted to determine potential demographic, clinical, and criminal profile differences between these two extreme groups. Significant differences included a greater presence of nonpsychotic minor diagnoses and number of previous hospitalizations for the probable malingering group. Trends toward differences were noted for higher use of psychotropic medications and more previous contact with mental health services for the probable malingering group. 


\section{Acknowledgements}

I would like to thank Dr. Fremouw for being an excellent mentor and teacher throughout my graduate school training. While I appreciated your guidance and skills as a student, I continue to recognize how much you taught me with each day. I would also like to extend my gratitude to the other members of my dissertation defense committee, Dr. Larkin, Dr. Cohen, Dr. Mogge and Dr. Baker. All of you have significantly contributed to my studies and professional development, as committee members and as supervisors and teachers. Lastly and most importantly, I cannot thank my family enough for all of their love, support, and encouragement. I could not have completed this project or graduate school without my mother, father, brother, and grandparents. 
Table of Contents

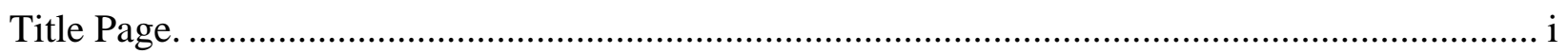

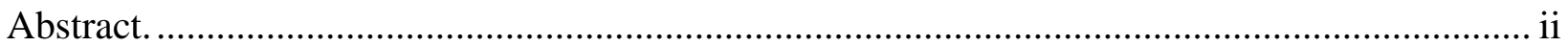

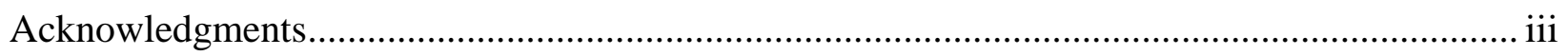

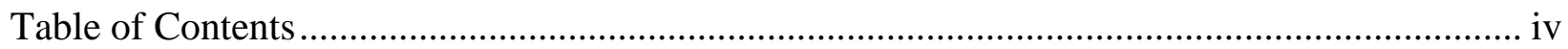

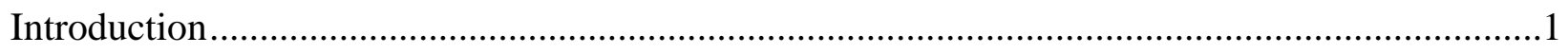

Assessments Used to Detect Feigned Mental Disorders.................................................3

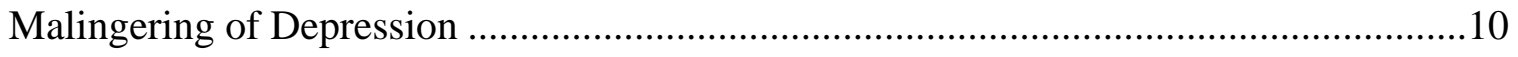

Clinical and Demographic Profile Differences......................................................17

Purposes / Aims of the Present Study …...................................................................18

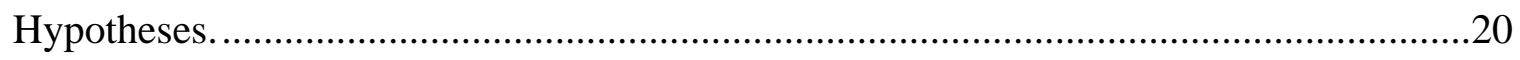

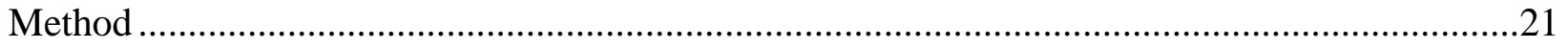

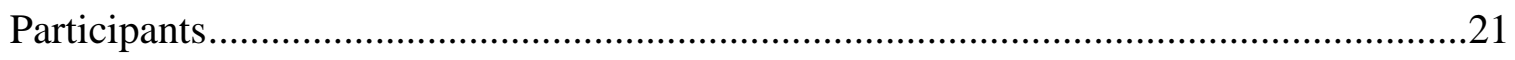

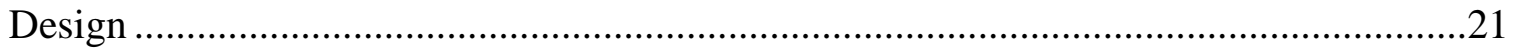

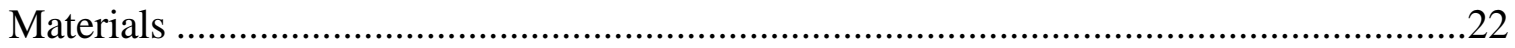

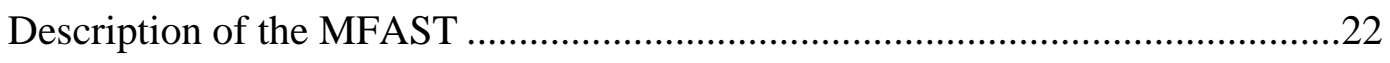

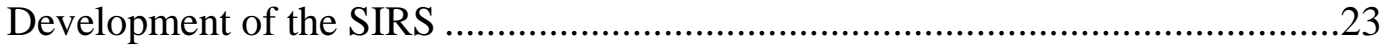

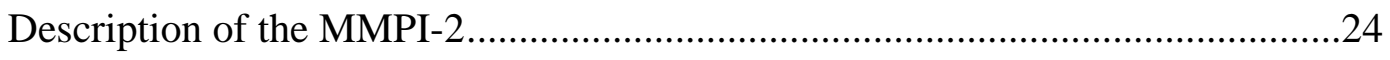

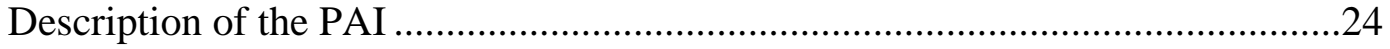

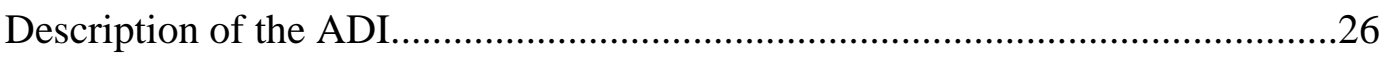

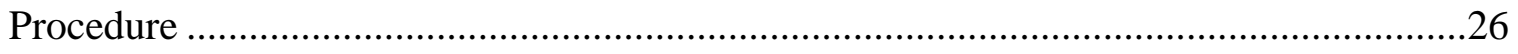

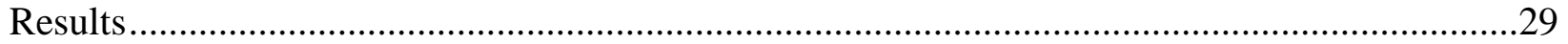




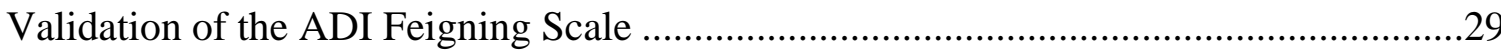

Demographic, Clinical, and Criminal Profile Differences..........................................30

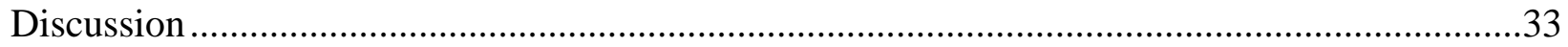

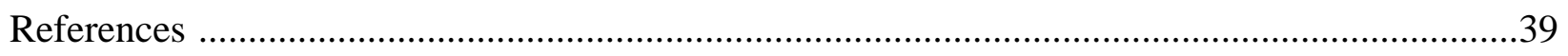

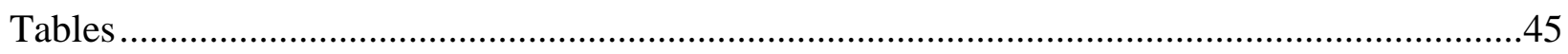

Table 1 (Comparison of Demographic Variables) ....................................................45

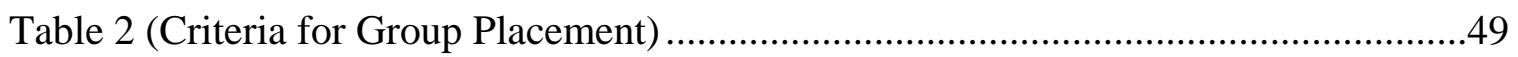

Table 3 (ADI Scale Scores Across Group Conditions) ..............................................50

Table 4 (Detection Rates of ADI Feigning Scale Scores) ..........................................51

Table 5 (Inter-rater Reliability for Variables) ..........................................................52

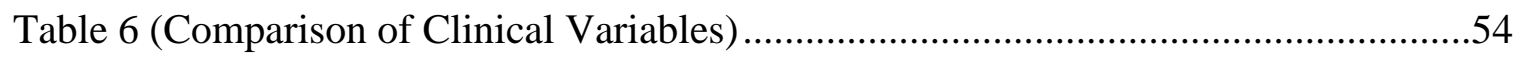

Table 7 (Comparison of Criminal / Legal Variables) ..................................................57

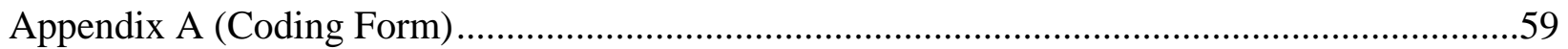


Validation of the Assessment of Depression Inventory (ADI) Feigning Scale and Clinical, Demographic, and Criminal Profile Differences between Probable Malingerers and Psychiatric Inpatients

In most clinical settings, the authenticity of an individual's presentation of symptoms is rarely questioned. However, psychologists working in forensic settings must consistently evaluate the veracity of information given to them. From feigning psychosis or depression to avoid prosecution of a crime, to claiming an anxiety disorder in order to receive disability assistance, people from all socioeconomic and educational backgrounds have been suspected of or found to be malingering (Rogers, 1997). The Diagnostic and Statistical Manual of Mental Disorders (DSM-IV-TR; American Psychiatric Association [APA], 2000) defines malingering as "the intentional production of false or grossly exaggerated physical or psychological symptoms, motivated by external incentives" (p. 739). The DSM-IV-TR (2000) also suggests that four factors should particularly alert clinicians to the possibility of malingering: "medicolegal context of presentation (e.g., the person is referred by an attorney to the clinician for examination), marked discrepancy between the person's claimed stress or disability and the objective findings, lack of cooperation during the diagnostic evaluation and in complying with the prescribed treatment regimen, and the presence of Antisocial Personality Disorder" (p. 739). While these guidelines offer factors which raise the question of malingering, clinicians are in general agreement that they do not offer enough specificity, clarity, and ease of application to be useful (Cunnien, 1997; Rogers, 1997).

Between $15-17 \%$ of cases presented in forensic settings have been estimated to involve malingering of symptoms (Rogers \& Bender, 2003). However, Norris and May (1998) estimated rates as high as $45 \%$ in a correctional setting. Among the general psychiatric population, several 
studies estimate rates to be approximately 7\% (Rogers, Sewell, \& Goldstein, 1994; Rogers \& Salekin, 1998). When considering both the financial and judicial implications of failing to identify those who are not mentally and physically disabled but are attempting to manipulate other individuals or the court, the importance of assessments to detect malingering is clear. Difficulties are inherent in the assessment of malingering and are multi-faceted. Differentiation must be made between malingering and factitious disorders (internally motivated feigned illness) and malingering and somatoform disorders (unintentional feigning of symptoms believed to be genuine to the individual) (APA, 2000). Among inpatient populations, where the presence of those with factitious or somatoform disorders is likely to be higher than in outpatient settings, these distinctions become crucial not only to detect false presentations of symptoms but also to successfully and effectively treat those with factitious or somatoform disorders.

Another difficulty is the limited number of measures available for the detection of specific disorders being feigned rather than simply the identification of deceptive response styles. Multi-scale personality measures that include validity index scores are not recommended by themselves to determine the presence of malingering, because as the testing manuals state, a variety of factors may contribute to elevated validity scales, including severe distress or psychosis (Butcher, Williams, Graham, Tellegen, \& Kaemer, 1989; Morey, 1991). A steadily growing body of research now exists supporting a multimodal assessment of malingering (Guriel et al., 2004; Resnick, 1997; Rogers \& Bender, 2003) that includes both general psychological measures and malingering-specific measures. Rogers and Bender (2003) also distinguish among the different types of malingering-specific measures and categorize these assessments into those that detect malingering of mental disorders and those that detect feigned cognitive impairment. Due to the present study's aims and purposes, only those measures used to detect malingering of 
mental disorders will be addressed. Rogers (1997) also suggests using a threshold model combined with a clinical decision-making model in the diagnosis of malingering which first involves identification of indicators of malingering during the evaluation procedure that then lead one to the use of specific measures to assess for malingering. Therefore, assumptions are not made at the beginning of the evaluation process but data is obtained based on each individual's presentation if known indicators of malingering are present. The data obtained for this study was based upon treatment team decisions that were made due to observations or assessment scores that indicated possible malingering. The malingering-specific measures were then administered after suspicions were raised rather than at the time of the intake.

\section{Assessments Used to Detect Feigned Mental Disorders}

Multi-scale self-report personality inventories are frequently used in assessing for the presence of deceptive response styles; the Minnesota Multiphasic Personality Inventory-2 (MMPI-2) and the Personality Assessment Inventory (PAI) are among the most popular (Rogers $\&$ Bender, 2003). Both measures benefit from validity scales that are designed to detect dishonest and/or random responding. The validity scales assist clinicians in determining questionable presentations of symptoms.

The MMPI-2 includes validity scales that measure atypical, deviant responding $(F$, Infrequency Scale), unrealistic positive responding ( $L$, Lie Scale), and a similar but more subtle correction scale ( $K$, Correction Scale) for socially desirable, positive responding (Groth-Marnat, 2003). In addition, the Psychopathology Infrequency Scale $(F p)$ has been found to be particularly helpful in identifying deceptive responding in simulated malingering research (Arbisi \& BenPorath, 1995). While not as well-known as the other validity scales, the $F p$ consists of 27 MMPI2 items infrequently endorsed by inpatients and normal controls. Arbisi and Ben-Porath (1995) 
suggest its use in conjunction with the other validity scales among populations and in settings that include high rates of psychopathology and psychological distress. Studies using the validity scales on the MMPI-2 to detect malingered PTSD have also found high rates of positive predictive power (Bury \& Bagby, 2002; Lewis, Simcox, \& Berry, 2002; Morel, 1998). In particular, scales used to assess inconsistent responding and symptom exaggeration appear to be effective at detecting possible deception (Fox, Gerson, \& Lees-Haley, 1995; Lewis et al., 2002).

Validity scales on the PAI used to assess malingering are the Negative Impression Management (NIM) scale, the Malingering Index (MAL; Morey, 1996), and the Rogers Discriminant Function (Rogers, Sewell, Morey, \& Ustad, 1996), the last two examining features of the entire PAI profile (Scragg, Bor, \& Mendham, 2000). Specificity of the PAI, or accuracy at correctly identifying honest responders (thus failing to misclassify honest responders as malingering), has been found to be high with the use of recommended cutoff scores (Liljequist, Kinder, \& Schinka, 1998; Scragg et al., 2000).

Three research methods are often employed when examining malingering with measures such as the MMPI-2 and PAI: simulation designs, known-groups comparisons, and differential prevalence designs (Rogers, 1997). Simulation designs are the most common and involve three groups: individuals without a disorder instructed to feign a particular disorder, controls chosen from the same nonclinical group told to respond honestly, and a clinical comparison group consisting of individuals who are diagnosed with the disorder in question. The simulation design has limitations in its generalizability; however, researchers can take steps to increase external validity (Rogers, 1997).

Known-groups comparisons use individuals in clinical settings who have been identified as suspected malingerers (Rogers, 1997). This group is then compared to individuals with the 
disorder in question who respond honestly. The benefit of using this design is its applicability to real-world settings and situations. Limitations to this method include correct classification of known groups and accessibility to such populations. The differential prevalence design is a more recent method that is the least methodologically sound (Rogers, 1997). Individuals who are assumed to vary in response style and motivation are grouped and then compared, and no other attempts are made to determine honest versus dishonest responders. For example, individuals who are involved in litigation are assumed to be more likely to malinger than individuals with the same condition who are not involved in litigation, so the two groups are compared on this basis alone (Rogers, 1997). Rogers (1997) cautions that this design does not allow an investigator to determine prevalence rates for those deliberately distorting symptoms, for identification of individuals who are or who are not malingering within each group, and the difference in performance between those in each group.

Most malingering research employs the simulation design, with few known-groups comparisons, which require the use of "gold standard" assessments to accurately identify and classify those who are likely malingering and those who have a legitimate diagnosis. "Gold standard" assessments have undergone extensive validation studies, and as a result of sound psychometric properties, they are used as a measure against which new assessments examine their validity. Examples include the Minnesota Multiphasic Personality Inventory-2 (MMPI-2), the Personality Assessment Inventory (PAI), and the Structured Interview of Reported Symptoms (SIRS).

Numerous studies have examined the ability of the MMPI-2 to detect deceptive response styles using simulation designs. Bury and Bagby (2002) asked participants to fake PTSD and found that those given information on the validity scales were the most successful at avoiding 
detection on the feigning scales, but that the $\mathrm{F}$ scales $(F, F(b)$, and $F(p))$ were all useful in detecting feigned responses, producing high rates of positive and negative predictive power. Walters and Clopton (2000) also used a simulation design to determine the effectiveness of the MMPI-2 at detecting feigned depression. Three hundred and seventy college students were told to malinger depression but were provided with different types of information to do so. One group received information on symptoms of depression, another on MMPI-2 validity scale and indexes information, and another received both symptom and validity scale information. A last group was given no information. MMPI-2 scores were compared among malingering groups and between honest controls. Unlike previous studies involving the feigning of schizophrenia or closed head injuries, the participants in this study who were given information about either the validity scales or symptoms of depression were most successful at avoiding detection. Other studies have found that participants given symptom information were not as successful as those given validity scale information. The results suggest that individuals who are attempting to malinger depression and have knowledge of either the symptoms of depression or of validity scales on the MMPI-2 could be successful at avoiding detection (Bury \& Bagby, 2002).

Rogers, Ornduff, and Sewell (1993) also used a simulation design to examine the effectiveness of the Negative Impression Management (NIM) scale of the PAI. The authors compared the responses of coached, or prepared, simulators from those of less prepared, or naïve, simulators. College students were asked to feign either schizophrenia, depression, or generalized anxiety disorder. Coached simulators were allowed one week to prepare for their task and were allowed use of any available resource with the exception of the PAI manual. Naïve simulators were provided with information on the symptoms of the specific disorder but only in the context of their instructions to feign the disorder. They were given no time to prepare or 
study. Results revealed that almost all of the simulators elevated the appropriate scale according to their disorder. The NIM scale's effectiveness at detecting feigned presentations varied according to the disorder. It was most effective at detecting feigned schizophrenia, marginally effective with feigned depression, and least effective with feigned generalized anxiety disorder (Rogers, Ornduff, \& Sewell, 1993). Interestingly, no differences between prepared and unprepared simulators were found except for those simulating depression, in that those with one week's preparation obtained more elevated depression scale scores and lower NIM scores, calling into question the usefulness of the PAI NIM scale at detecting feigned depression in particular.

In addition to the use of multi-scale self-report personality inventories, recent advances in the assessment of malingering have led to a trend in the development of measures designed specifically to assess for deceptive responding, without measuring for the presence of pathology. A structured interview that has been developed is the Structured Interview of Reported Symptoms (SIRS; Rogers, Bagby, \& Dickens, 1992). Rogers et al. (1992) examined the effectiveness of the SIRS, the 172-item "gold standard" structured interview designed to assess deceptive response styles, in detecting malingered PTSD, schizophrenia, and mood disorders.(A more detailed explanation of the SIRS scales is provided in the Materials section below.) Rogers, Gillis, Bagby, and Monteiro (1991), using coached and uncoached simulators, found that while coached simulators were able to alter their responses on the SIRS scales and decrease their scores, the SIRS was still able to distinguish the simulators from the legitimate presentations of psychiatric inpatients $(96.7 \%$ of inpatients were identified as legitimate and $84.6 \%$ of simulators were correctly identified). In this study, participants were not only provided with information on psychiatric disorders but also with guidelines for how to avoid detection on assessment 
measures. Only one SIRS scale, Inconsistency of Symptoms, failed to reveal differences between the malingering groups and honest controls.

Rogers, Kropp, Bagby, and Dickens (1992) examined the effectiveness of the SIRS using participants in a correctional setting told to simulate one of three specific disorders: schizophrenia, mood disorders, and PTSD. These participants were provided with information from the DSM-III-R for the particular disorder that they were asked to feign. Inpatient psychiatric residents were also given the SIRS as part of their intake assessment and asked to respond honestly. Significant differences were found between correctional participants and the inpatient residents considered to be presenting honestly. Eight out of the twelve SIRS scales were consistently able to distinguish feigners from legitimate presentations. The Symptom Onset scale did not differentiate any of the malingering groups from psychiatric inpatients. The other three scales that did not distinguish simulators from controls (Overly Specified Symptoms, Inconsistency of Symptoms, and Reported vs. Observed Symptoms) failed to do so only for the group feigning PTSD. Rogers et al. (1992) state that "even though the SIRS was not designed to measure specific syndromes (e.g., schizophrenia), we found it interesting that some differences did occur as a result of which specific disorder was feigned" (p. 647). While this study lends credibility to the SIRS at detecting feigned presentations, of note is that the feigning of specific disorders does present unique presentations and possibly the need for different detection strategies.

Several screening instruments that are less time consuming and require less training to administer have also been developed. The Miller Forensic Assessment of Symptoms Test (MFAST; Miller, 2001), based upon the deceptive response strategies used by the SIRS, is a brief, 25-item structured interview that assesses malingering of psychotic symptoms based on overall 
response style. Initial studies have found that the M-FAST is successful at detecting malingering using known-groups comparisons. Outpatient disability claimants who were suspected of malingering based on elevated SIRS scores had significantly higher total M-FAST scores than honest responders (Miller, Guy, \& Davila, 2000). In a separate study, malingerers from a maximum-security penitentiary were identified by elevated SIRS scores and also received significantly higher M-FAST scores than a group of prisoners without elevated SIRS scores who were considered to be honest responders (Guy \& Miller, 2000).

In addition, the Structured Inventory of Malingered Symptomatology (SIMS; Widows \& Smith, 2005) is a 75-item self-administered screening measure designed to detect malingering of "feigned psychopathology and cognitive function" (p. 4). Subscales of the SIMS include Psychosis, Neurologic Impairment, Amnestic Disorders, Low Intelligence, and Affective Disorders. Smith and Burger (1997), using a simulation design with college students, examined the validity of the SIMS compared to the $F$ and $K$ sales of the MMPI, and portions of the malingering scale. The college students were instructed to feign one of seven specific psychiatric conditions (psychosis, amnesia, neurologic impairment, mania, depression, low intelligence, or "fake bad") or answer honestly. The authors found that the SIMS total score, not specific subscale scales, had a higher sensitivity rating $(95.6 \%)$ than the other validity indices. Unlike most measures of malingering, the SIMS attempts to combine the two major areas of malingered mental disorders and feigned cognitive function mentioned by Rogers and Bender (2003). While the breadth of the SIMS in detection of feigned symptom presentation is unique, an obvious limitation concerns its use in identifying what specific disorder an individual is attempting to feign. Smith and Burger's (1997) finding that the total score, rather than specific subscale scores, led to the highest sensitivity rating despite participants' instructions to feign a specific disorder 
emphasizes this lack of clarity. Multiple scale elevations could represent an attempt to feign several disorders; however, this lack of specificity may prove unhelpful to clinicians in inpatient or outpatient settings attempting to determine proper treatment needs or answer certain legal questions, such as disability qualification, competency to stand trial, criminal responsibility, or future risk.

Advances have been made in designing measures to specifically detect for the presence of a psychiatric disorder along with feigning subscales; however, this progress has primarily focused on posttraumatic stress disorder and cognitive or intellectual impairment. The Trauma Symptom Inventory (TSI; Briere, 1995) and the Detailed Assessment of Posttraumatic Stress (DAPS; Briere, 2001) are two PTSD-specific measures that contain validity scales to assess atypical responding and possible malingering and also the presence and severity of PTSD. The Morel Emotional Numbing Test (MENT; Morel, 1998) and the Morel Emotional Numbing TestRevised (MENT-R; Messer \& Fremouw, 2007) have been designed to detect feigned PTSD but do not assess for the presence of the disorder.

\section{Malingering of Depression}

While an array of measures have been designed to detect deceptive response styles, either among personality inventories or malingering specifically, an area of research that has been left largely unexplored is the development of assessments designed to detect the malingering of depression. In social security disability evaluations, large sums of money are often at stake when persons are found unable to work due to depression. In criminal trials, possible reduced sentences, lesser charges, or findings that one is not criminally responsible because of depression have dramatic implications for justice and fairness in the United States legal system. In addition, psychiatric hospitals expend large amounts of money in the treatment of patients with a 
multitude of psychopathology, and individuals feigning depression use resources necessary in treating patients with genuine impairment.

Major Depressive Disorder, as defined by the DSM-IV-TR (APA, 2000), is a mood disorder with multiple specifiers, such as "mild, moderate, severe without psychotic features, severe with psychotic features, chronic, with catatonic features, with melancholic features, with atypical features, and with postpartum onset" (p. 370). In addition, Major Depressive Disorder must be distinguished as either a single episode or recurrent. Five of nine symptoms must be present for two weeks. The nine symptoms in the DSM-IV-TR (2000) include depressed mood, loss of interest or pleasure in activities, weight loss or gain, insomnia or hypersomnia, psychomotor agitation or retardation, fatigue or loss of energy, feelings of worthlessness and guilt, indecisiveness or lack of concentration, and recurrent thoughts of death or suicidal ideation. Rehm, Mehta, and Dodrill (2001) mention that affective states of depressed individuals may be "flat" and may involve anxiety, anger, or irritability.

The identification and treatment of Major Depressive Disorder has dramatic clinical implications, as this disorder is associated with high mortality rates. The DSM-IV-TR (APA, 2000) states that "up to $15 \%$ of individuals with severe Major Depressive Disorder die by suicide" (p. 371). Individuals over the age of 55 with Major Depressive Disorder have a fourfold increase in death rates, and individuals in general medical settings with Major Depressive Disorder are likely to have more pain and physical illness and less ability to function physically and socially. Lifetime risk for Major Depressive Disorder in the adult population varies from $10 \%$ to $25 \%$ for women and $5 \%$ to $12 \%$ for men (APA, 2000). Point prevalence rates in adult community samples, or the percentage of individuals who experience Major Depressive Disorder within a year, range from 5\% to $9 \%$ for women and from $2 \%$ to $3 \%$ for men. Females have been 
found to attempt suicide more frequently than males; however, males are much more likely to complete suicide (Fremouw, dePerczel, \& Ellis, 1990). This disorder has not been found to be associated with ethnicity, education, income, or marital status.

The importance and necessity of distinguishing genuine from feigned major depression is clear in that failing to identify legitimate depression can be lethal to the individual and lead to major impairment in life functioning; however, failing to identify those who are feigning depression can lead to economic, social, and legal ramifications to society. Difficulties with using multi-scale personality inventories solely to identify malingered depression have been mentioned above in that the validity scales reflect overall response style and may not necessarily indicate malingering. Malingering-specific measures such as the SIRS and M-FAST are not designed to address the malingering of specific disorders; thus, these measures broadly assess for a variety of malingered psychopathology not specific to depression. Furthermore, interviews such as the SIRS are time-consuming (45 to 75 minutes) and require significant training on the part of the administrator.

Steffan, Clopton, and Morgan (2003) developed a validity scale for the MMPI-2 to specifically detect malingered depression, the Malingered Depression $(M d)$ scale. The scale was designed using 32 items that differentiated college students told to feign depression from controls diagnosed with depression. The college student simulators were provided information about both the symptoms of depression and MMPI-2 validity scales. A follow-up study using the same design found promising results. Bagby, Marshall, and Bacchiochi (2005) examined the validity of the $M d$ scale as compared to MMPI-2 F scales in effectiveness at detecting feigned depression. Twenty-three MMPI-2 protocols, completed by mental health professionals told to feign depression, were obtained from a data base at a university depression clinic. Another 225 
MMPI-2 protocols were obtained from a psychiatric facility and were selected based on patients meeting criteria for major depression and not meeting criteria for other Axis I disorders or an Axis II disorder. Results indicated that the $F$ scales $(F, F b$, and $F p)$ and the $M d$ scale distinguished the experts feigning depression from the honest controls. The $M d$ scale added some incremental validity to the F scales in detection of feigned depression; however, it was not able to distinguish many more individual cases as compared to $F b$ and an $F / F p$ combination. While the $M d$ scale demonstrated minimal improvement over the F scales in the detection of malingered depression, its improvement was not robust, despite the $F$ scales being based on overall response style versus feigning of a specific disorder.

The SIMS (Widows \& Smith, 2005) includes a subscale for affective disorders that "assesses the degree to which a respondent reports atypical symptoms of depression and anxiety" (p. 4). However, a major disadvantage of the SIMS, and other malingering-specific measures, is the failure to assess for the presence of the pathology in question, and total scores have been found more useful in detecting malingering than specific subscales. While a broad range of disorders are included, the measure lacks specificity for Major Depressive Disorder.

Bracken and Howell (2004) published the Clinical Assessment of Depression (CAD), a self-report 50-item assessment of depression for individuals aged 8 to 79 years of age. The authors suggest that strengths of the CAD are its ability to be used across a wide range of ages, in that it includes items specific or unique to the expression of depressive symptoms across ages. The CAD also includes a total scale score, subscale scores, validity scales, and critical item clusters. Subscales include Depressed Mood (DM), Anxiety/Worry (AW), Diminished Interest (DI), and Cognitive and Physical Fatigue (CPF). Validity scales include a Negative Impression Score (NI), and Inconsistency Score (IN), and an Infrequency Score (IF). Critical item clusters 
include Hopelessness, Self-Devaluation, Sleep/Fatigue, Failure, Worry, and Nervous). Bracken and Howell (2004) include in the CAD manual a chapter on the development and standardization of the $\mathrm{CAD}$, including norming procedures and derivation of $T$ scores. However, all reliability and validity information is provided by the authors in the manual and has not been published in any study to date with outside researchers. The authors indicate that "content validity of the CAD was ensured during the scale development by writing items based on known characteristics of depressed individuals and symptomatic criteria found in such diagnostic methodology as the DSM-IV' (p. 43). Bracken and Howell (2004) examined construct validity in an independent validity sample with the BDI-II and RADS (Reynolds Assessment of Depression Inventory), and using total scores, found a .77 correlation with the BDI-II and a .88 correlation with the RADS. Studies examining the effectiveness of the validity scales have not been conducted or published thus far, representing a major limitation of this measure.

Recently, Mogge and LePage (2004) designed the Assessment of Depression Inventory (ADI), an instrument that measures not only for presence and severity level of depression, but also includes scales which measure feigned and random responding. Several brief, self-report measures exist which measure the severity and presence of depression, such as the Beck Depression Inventory - II (BDI-II; Beck et al., 1996) and the Geriatric Depression Scale (GDS; Koenig et al., 1998). The strengths of these measures include their brevity, ease of scoring, and their capacity for multiple administrations at different points in time to assess for lessening or worsening of severity levels. However, all of these measures are face valid, making them particularly vulnerable to distortion, as an individual attempting to feign depression may simply respond in the extreme to each question. These measures also do not include a scale to assess for random responding, in which an individual who is uncooperative with the assessment may 
simply not read the items.

Mogge and LePage (2004) state that "the goals of the ADI are to provide a brief selfreport assessment of legitimate depressive symptoms. It also has two validity scales embedded within it. One can be used as a screening tool for detecting those who may be attempting to exaggerate depression; the other addresses the detection of random or careless responding" ( $p$. 108). The ADI has been validated using a sample of psychiatric inpatients instructed to respond honestly and two different types of simulators instructed to feign depression to get out of trouble with the law: one group of community residents considered naïve to symptoms of depression and assessment techniques, and another group comprised of staff from the hospital who work with inpatients (psychiatric aids, social workers, or nurses), considered more sophisticated in their knowledge of mental illness (Mogge \& LePage, 2004). Results indicated significant differences between the honest responders and those asked to feign depression, with no differences found between the two groups of feigners (naïve and sophisticated). With the honest sample, the Depression scale correlated with the BDI-II at .90. Over 79\% of the feigners (combined into one group) were identified using the Feigning scale of the ADI with a cut-score of 15, indicating that a score of 15 detected a large portion of simulated malingerers while keeping false positive rates low (less than 3\% in combined patient samples).

A second study (Mogge, 2006), using an inpatient sample of 89 participants, compared the ADI Depression scale scores (DEP) to the Depression (DEP) scale of the Personality Assessment Inventory (PAI) and its three subscales (DEP cognitive, DEP affective, and DEP physical). In addition, the ADI Feigning Scale (Fg) was compared to the four validity scales of the PAI, the NIM and MAL Index (which detect negative bias) and the Positive Impression Management scale (PIM) and Defensiveness Index (DEF). Pearson Product Moment 
Correlations revealed significant correlations (at the .01 level) among the ADI Depression Scale and all four Depression scales of the PAI, with the strongest correlation found with the PAI's DEP affective subscale $(r=.81)$. Other scale correlations ranged from $r=.64$ to $r=.78$. PPM Correlations were also significant in expected directions for the ADI Feigning scales and all four validity scales of the PAI. The ADI Fg scale correlated positively with the NIM $(r=.70)$ and MAL $(r=.69)$ and negatively with the PIM $(r=-.44)$ and DEF $(r=-.33)$. This study also examined cut-scores for the Feigning scale against the PAI by using the NIM and MAL scores to identify probable malingerers. Although only 15 participants were identified as purposefully feigning, a ROC area under the curve was found at $80 \%$ of probable malingerers with a cut-score of 15 and $85.3 \%$ with a cut-score of 14 .

In continuing to explore the validity of the ADI Depression scale and Feigning scale with additional populations and settings, Mogge, Steinberg, Fremouw, and Strunk (in press) compared the ADI DEP scale to three other measures of depression, the Beck Depression Inventory - II (BDI-II), the Zung Self-Rating Depression Scale (ZSDS), and the Personality Assessment Inventory (PAI) with an outpatient sample of psychiatric patients. Pearson Product Moment Correlations among all four Depression scales were significant (between $\mathrm{r}=.81$ and $\mathrm{r}=.82$ ). Using the PAI Depression scale, BDI-II, and ZSDS, groups were classified as being moderately depressed if two of the three scales were above cut-scores for depression and not depressed (controls) if less than two or none of the three Depression scales exceeded the cut off. An ADI Depression scale cut-score of 39 was used and Receiver Operating Characteristics were examined. Sensitivity rates were found to be .96 , with a specificity of .89 , and an overall hit rate of .94. Positive predictive power was .96, while negative predictive power was .89. Correlations between the ADI Feigning $(\mathrm{Fg})$ scale and the PAI validity scales were not as strong as expected, 
but all were significant at the .01 level. The Fg scale of the ADI was positively correlated with the NIM $(r=.59)$ and MAL $(r=.33)$ and negatively correlated with the PIM $(r=-.33)$ and DEF $(\mathrm{r}=-.35)$. While validation of feigning scales is necessary in the advancement of malingering assessment techniques, the examination of possible clinical and demographic differences between deceptive and honest responders may also prove useful, particularly when the threshold model (Rogers, 1997) is employed in the assessment of malingering.

\section{Clinical and Demographic Profile Differences}

Another relatively novel area of research regarding forensic populations is the examination of profile differences between known groups affecting the legal system. Studies thus far have focused on attempting to find differences between defendants predicted restorable for competency to stand trial, and those found not restorable for competency to stand trial (Hubbard \& Zapf, 2003; Hubbard, Zapf, \& Ronan, 2003). Vess, Murphy, and Arkowitz (2004) examined clinical and demographic differences between sexually violent predators and other committed forensic patients in a hospital setting. One major aim of finding differences, if they exist, is to assist examiners in more accurately determining if these individuals can or cannot be restorable to competency or if particular offenders are more dangerous and require unique treatment planning. The finding of these studies do not pertain to the aims of the present study; however, the methodology is relevant in that only one other study has been found that briefly examined demographic and clinical comparisons between suspected malingerers and an honest group of forensic patients.

Lewis, Simcox, and Berry (2002) conducted a study using a forensic sample of 55 males undergoing competency to stand trial evaluations or criminal responsibility evaluations with the primary aim of examining the effectiveness of the SIMS and selected MMPI-2 validity scales in 
identifying feigned psychiatric symptoms with the SIRS as the criterion measure for group placement. The authors included in their results section information regarding differences between the feigning $(n=24)$ and honest $(n=31)$ groups. Descriptive statistics and chi-square analyses were used to find significant differences. However, the authors did not systematically detail the methodology behind obtaining this information. Findings included that feigners were on average younger than the honest responders, the presence of "strong trends" toward differences on ethnicity and intelligence, and no differences on any legal variable except the feigning group faced potentially longer sentences. The authors do indicate that Axis I and Axis II diagnoses were taken from clinicians' reports; however, it is unclear if these reports were verbal or found in charts and if they were current or historic. The number of individuals given an Axis I diagnosis did not differ significantly between the two groups; however, more feigners were given an Axis II diagnosis than honest responders, and of those receiving an Axis II diagnosis, more feigners received a diagnosis of Antisocial Personality Disorder. Other analyses were conducted using the MMPI-2 clinical and validity scales in the prediction of group placement (Lewis, Simcox, \& Berry, 2002). A brief paragraph in the conclusion mentions the factors identified as being more associated with belonging to the feigning group; however, the authors do not provide a rationale or explanation for this minor addition to their study.

\section{Purposes / Aims of the Present Study}

Purpose One

The first purpose of the study was to validate the Assessment of Depression Inventory (ADI) Feigning Scale using "gold standard" assessment measures to determine group placement of psychiatric inpatients in a known-groups research design. Archival data was collected to determine categorical placement into one of three groups: non-malingering inpatients, probable 
malingerers, and indeterminate malingerers. All of the inpatients received this battery of assessments based on Rogers (1997) recommended threshold and clinical decision making model of malingering in that only patients suspected of feigning received the full battery of malingering-specific measures (based on clinical observation, indicators present in clinical interviews, or assessment data), rather than as a part of routine intake procedures. The identification of these three groups was used to validate the Assessment of Depression Inventory (ADI) Feigning Scale with a known group of malingering inpatients, which provides more applicability to real-world settings and situations than simulation designs. Due to the use of dual criteria using both "gold standard" personality inventories and a malingering measure (the SIRS), correct classification of these groups is strengthened. This is the first study to use dual criteria "gold standard" measures for group placement.

A second aim of the present study was to identify potential demographic, clinical, and criminal variables that distinguish between probable malingerers and honest psychiatric inpatients. The research method used involved systematically reviewing chart data from two groups of individuals classified as either probable malingerers or honest responders. This purpose was similar to the studies with restorable and non-restorable defendants, in that data was examined that could assist examiners in determining feigned from honest presentations of symptoms. This data was not intended to be used solely in that determination or to bias or stereotype particular individuals, but to be used in conjunction with Rogers and Bender's (2003) detailed suggestions of using multi-modal, multi-assessment strategies along with extensive review of an individual's history, the use of secondary sources of information, and threshold and clinical decision making strategies. Hubbard and Zapf (2003) and Hubbard, Zapf, and Ronan (2003) provide extensive detail in the methodology used to accurately compare the two known- 
groups samples and the statistics used to find differences. Their procedures were followed in the present study and are described below and in the Methods section.

In order to accomplish the aims of the present study, only the two extreme groups, probable malingerers and honest psychiatric inpatients, were used when analyzing data. This decision was based on several reasons. First, this study is unique in that dual criteria were required for group membership, a high standard intended to improve the accuracy of the knowngroups sample. This standard would be "watered down" with the inclusion of the indeterminate group, in that by definition this group did not meet dual criteria, similar to the rationale used by Lewis, Simcox, and Berry (2002) when eliminating an indeterminate group of malingerers. As such, results of the study would be less meaningful if the indeterminate group had been collapsed into the honest group. As Rogers (1997) points out, a strength of using a known-groups design is the increased applicability to real world settings and situations, a strength that would have been weakened if one of the known groups did not have to meet the dual criteria standard; therefore, all subsequent analyses used only the groups which met this standard.

\section{Hypthotheses}

1. The ADI Feigning Scale (Fg) has not been validated with a known-groups sample using dual criteria standards of a personality measure and the SIRS. However, based on previous studies of the Fg scale (Mogge, 2006; Mogge \& LePage, 2004; Mogge, Steinberg, Fremouw, \& Strunk, in press), it was hypothesized that the Fg scale would be effective at differentiating the groups and have at least moderately strong detection rates between the honest psychiatric inpatients and probable malingerers, including sensitivity, specificity, positive predictive power, negative predictive power, hit rate, and receiver operating curve (ROC) rates.

2. Limited studies have examined demographic, clinical, and criminal profile differences within 
forensic populations, and only one study has included any information on potential differences between probable malingerers and honest responders (Lewis, Simcox, \& Berry, 2002). Based on this study and the four factors listed in the DSM-IV-TR (APA, 2000) to alert clinicians to the possibility of malingering, it is hypothesized that probable malingerers may have more notable criminal histories and more Axis II diagnoses. Data will be coded categorically, and chi-square analyses as well as descriptive statistics will be used to compare the data.

\section{Method}

\section{Participants}

The participant database was obtained from archival data collected from 2004 to 2006 at William R. Sharpe, Jr. Hospital in Weston, WV, an inpatient facility with four units for civilly committed patients and two units for forensic patients. For the 2004-2005 fiscal year, the average daily census at the hospital was 152 , with an average of 81.25 admissions per month and an average of 81.67 discharges per month. For the 2005-2006 fiscal year, the average daily census was 153 , with an average of 88.42 admissions per month and an average of 87.17 discharges per month. Approval for conducting the research was granted by the university Institutional Review Board and the hospital's research committee. The data was collected as either part of routine intake procedures or as deemed appropriate by treatment teams due to questions regarding the credibility of an individual's presentation.

\section{Design}

Seventy participants received the necessary assessment battery to be included in the study, and based on criteria described in the procedure section, were placed in one of three categories: non-malingering inpatients $(n=22)$, probable malingerers $(n=35)$, and indeterminate malingerers $(n=13)$. Among all participants, the average age was 34.2 years; $90 \%$ 
were male, and 10\% were female. Most of the participants were Caucasian (95.7\%); however, other races of participants included African American (2.9\%) and Other (1.4\%). Race, gender, age, education, marital status, education, living situation, employment and source of income demographics for the two extreme groups are summarized in Table 1. The indeterminate malingering group was excluded from analyses due to potential confounds that could be introduced by not meeting dual criteria standards for group membership. Materials

Due to the nature of the psychological assessment process at a large, inpatient hospital, not all participants received all of the measures listed below. However, each participant received either or both the Personality Assessment Inventory (PAI) or Minnesota Multiphasic Personality Inventory 2 (MMPI-2), both of which are well-established, multi-scale personality inventories with validity indices. In addition, patients may have received a Miller Forensic Assessment of Symptoms Test (M-FAST), a screening instrument for malingering, and not received a Structured Interview of Reported Symptoms (SIRS) due to not obtaining an elevated M-FAST score, which renders further malingering assessments unnecessary. Only participants who obtained a valid personality inventory profile and no elevation on the MFAST did not receive a SIRS, thus meeting criteria for non-malingering inpatient. All participants in either the indeterminate malingering group or probable malingering group received both gold-standard measures, the SIRS and either the MMPI-2 or PAI, in order to be included in the study. Among all participants, 65 were administered the PAI, and 14 received the MMPI-2. Nine participants completed both personality inventories.

Description of the Miller Forensic Assessment of Symptoms Test (M-FAST). This brief, structured interview contains 25 items and is designed as a screening measure for malingering 
based on an individual's response style. The M-FAST contains seven subscale scores and an overall total score. The seven subscales are based on strategies outlined by Rogers, Bagby, and Dickens (1992) in the creation of the SIRS: reported versus observed symptoms, extreme symptoms, rare combinations, unusual hallucinations, unusual symptom course, negative image, and suggestibility. A cut-off total score of six has been used to suggest dishonest responding, and research using the M-FAST has found this cut score to be most effective (Jackson, Rogers, \& Sewell, 2005; Miller, Guy, \& Davila, 2000; Miller, 2001). Alpha coefficients range from .44 to .82 for subscales and .92 for total score (Miller, 2001).

Description of the Structured Interview of Reported Symptoms (SIRS). This structure interview must be administered by a trained clinician and is designed to assess the presence of feigned psychiatric disorders. The SIRS consists of eight primary scales and includes both general and detailed inquires. The eight scales include: Rare Symptoms (RS), Symptom Combinations (SC), Improbable and Absurd Symptoms (IA), Reported vs. Observed Symptoms (RO), Direct Appraisal of Honesty (DA), Defensive Symptoms (DS), Overly Specified Symptoms (OS), and Symptom Onset and Resolution (SO) (Rogers, Bagby, \& Dickens, 1992). Based on normative data, each primary scale is classified into one of four categories: honest, indeterminate, probable feigning, and definite feigning. Typically, results are based on the number of scales classified as either probable or definite feigning. If one or more scales are classified as "definite," or if three or more primary scales are classified as "probable," or if the total raw score is above 76, then the individual is classified as feigning (Rogers, Bagby, \& Dickens, 1992). If six or more primary scales are in the "honest" range, the individual is determined to be an honest responder. Numerous studies have examined the validity and reliability of the SIRS. The SIRS manual provides data that using the above criteria as cut-offs, a 
positive predictive power of .98 has been found.

Description of the Minnesota Multiphasic Personality Inventory 2 (MMPI-2). Lees-Haley (2002) reports that the MMPI-2 is the most commonly used forensic evaluation instrument. This multi-scale, self-administered personality inventory consists of 567 true-false items. The MMPI2 contains 10 clinical scales as well as 3 standard validity scales and multiple additional validity indices. The most commonly used scales, found to be the effective, for the detection of feigning, or faking bad, are $F, F b$, and Infrequency minus Correction $(F-K)$, and $F(p)$. A meta-analyses by Rogers et al. (1994) found significant effect sizes with the $F$ and $F-K$ validity scales (Cohen's d > 2.00) when distinguishing honest and feigning participants. Rogers et al. (1994) suggested cut scores of $F>23(\mathrm{~T}=107)$ (hit rate of $87 \%), F b>16(\mathrm{~T}=108)$ (hit rate of $81 \%)$, and $F-K>10$ (hit rate of $85 \%$ ). The $F(p)$ scale was not covered in the meta-analysis but has been found by Arbisi and Ben-Porath (1998) to be superior to the $F$ scale. Using an $F(p)>$ 100(T), a hit rate of $97-98 \%$ was obtained.

Description of the Personality Assessment Inventory (PAI). This multi-scale, selfadministered inventory is also widely used in both forensic and non-forensic settings. Its focus is on the assessment of multiple clinical and personality variables. The measure consists of 344 items scored on a 4-point scale ("totally false," "slightly true," "mainly true," and "very true"). There are 22 nonoverlapping scales with 4 validity scales, 11 clinical scales, 2 scales assessing interpersonal style, and 5 scales related to treatment (Morey, 1991). The four validity scales consist of the Inconsistency Scale, Infrequency Scale, Negative Impression Management Scale, and Positive Impression Management Scale. Numerous supplemental validity indices have been developed in recent years in order to detect biased or distorted response style. To detect malingering, three scales have been primarily used: the Negative Impression Management (NIM) 
scale, the Malingering Index (Morey, 1996), and the Rogers Discriminant Function (RDF; Rogers et al., 1996).

The NIM consists of nine items and measure endorsement of "extremely bizarre and unlikely symptoms" (Bagby et al., 2002, p. 72) that may reflect an individual's attempt to exaggerate or create a negative impression of one's clinical status.

The Malingering Index, described by Bagby et al. (2002), has eight scoring criteria that were based on various PAI scales and subscales. The eight features of the Malingering Index were designed after observing PAI profiles of participants instructed to malinger a mental disorder and comparing those to clinical inpatients. Bagby et al. (2002) explain that the MAL Index "is designed to detect a response style that is characterized by over-endorsement of items that appear blatantly pathological to the general public, over-endorsement of items rarely endorsed by clinical populations, and under-endorsement of subtle symptoms and treatmentseeking items" (p. 72).

The Rogers Discriminant Function was designed by differentiating PAI profiles of honest patients from those told to feign mental disorders. Honest psychiatric inpatients' PAI profiles were distinguished from coached and uncoached individuals instructed to feign a mental disorder by using a discriminant function analysis (Roger et al., 1996). The individual's overall response pattern is compared to genuine inpatients' patterns, as it is assumed that those attempting to feign a disorder will not be capable of creating profiles similar to genuine inpatients.

Morey (1991) has examined the NIM's effectiveness using college students and found that those instructed to respond as if feigning a mental disorder score significantly higher than those instructed to respond honestly. In 1993, Morey used these profiles from college students to construct the 8-item Malingering Index. In a paper presentation, Gaies and Kinder (1995) 
examined the Malingering Index using honest controls, clinical patients, and simulated malingerers and found that no group scored positively for all 8 features; however, none of the honest controls or clinical patients scored greater than 3 on the index, indicating that scores greater than 3 would not have included any false positives (as cited in Liljequist et al., 1998). In a study comparing both the MMPI-2 and PAI validity scales and indices to detect simulated feigning of mental disorders (coached and uncoached), Bagby et al. (2002) found that the Rogers Discriminant Function (RDF) was "marginally superior" (p. 70) to the MMPI's $F$ and $F(p)$ in differentiating genuine psychiatric inpatients' profiles from those instructed to malinger.

Description of the Assessment of Depression Inventory (ADI). The ADI is a 39-tem selfreport measure that assesses the presence and severity of depressive symptoms. Individuals rate on a 4-point scale the frequency of experiences related to each item description in the past two weeks ("never," "some," "often," or "always.") In addition to the 19-item Depression (DEP) scale, there are two validity scales, including an 8-item Feigning (Fg) scale and 4-item Random (Rd) scale. Eight items are included as distracters. Mogge and LePage (2004) found that in a study using an honest inpatient control sample and a sample of participants instructed to feign depression, the Depression scale correlated with the BDI-II at .90. Over $79 \%$ of the feigners were identified using the Feigning scale of the ADI with a cut-score of 15, indicating that a score of 15 detected a large portion of simulated malingerers while keeping false positive rates low (less than $3 \%$ in combined patient samples).

In addition to the above measures, patient charts from William R. Sharpe, Jr. Hospital were be coded by one or two of three raters, blind to group membership, and reviewed to obtain information outlined below in the Procedure section.

Procedure 
Using archival assessment data collected from 2004 to 2006, inpatients who received either the MMPI-2 and/or PAI, the M-FAST and/or SIRS, and the ADI were grouped into three categories based on validity indices' cut-scores and criteria found to be most effective in the literature on the MMPI-2 (Arbisi \& Ben-Porath, 1998; Rogers et al., 1994) and the PAI (Bagby et al., 2002; Morey, 1991; Morey, 1996). In order to be considered effective, the criteria demonstrated adequate hit rates, and good sensitivity, specificity, and positive and negative predictive power.

The groups included: non-malingering inpatients, probable malingerers, and indeterminate malingerers. Classification criteria is summarized in Table 2. In order to be classified as a non-malingering inpatient, an individual would have received a total score of less than six on the M-FAST, thus making the SIRS unnecessary to administer, nonelevated validity indices on either the MMPI-2 or PAI, and elevated clinical scales on one of the two multi-scale personality inventories. In order to be classified as a probable malingerer, a patient would have received a total score of greater than 6 on the M-FAST, at least one primary scale of the SIRS in the "definite" range, or at least three primary scales in the "probable" range. In addition, the individual would have received a NIM $T$ score equal to or greater than 92 (two standard deviations above the clinical population mean and more stringent than the recommended 85) and a score of 3 or greater on the Malingering Index of the Personality Assessment Inventory (PAI), or meet any of the three criteria set by Rogers et al. (1994) in his meta-analysis of the MMPI-2 and one criteria found by Arbisi and Ben-Porath (1998) to achieve high hit rates for the MMPI-2: 1.) $F$ scale score $=$ or $>23(T$ score 107 or above $), 2$.) $F b$ scale score of 16 ( $T$ score of 108 or above), 3.) $F-K=$ or $>10$, and 4.) $F(p)$ score $=$ or $>100 T$. If any of these scores were elevated, individuals were placed in the probable malingering range as long as he/she also had an elevated 
SIRS score. In order to be classified as an indefinite malingerer, a patient would have received an elevated M-FAST and SIRS score (based on above criteria) or any of the elevated validity indices described above on either the MMPI- 2 or PAI, but not on both. Of note is that in all previous studies using known-group comparisons, only one criterion is used for group placement, such as the SIRS or MMPI-2. This study attempted to define groups more stringently by using two criterion measures for group placement.

The Assessment of Depression Inventory (ADI) Feigning scale was validated by comparing detection rates for the Feigning scale for the two extreme groups. Detection rates included information on specificity and sensitivity of the ADI feigning scale, positive predictive power, negative predictive power, and hit rate. An ANOVA was also conducted comparing ADI feigning scale scores between the extreme groups. Only these two groups were compared when examining demographic and clinical differences using the chart coding procedure described below.

In addition to validating the ADI Feigning scale, chart reviews were conducted using the non-malingering inpatients and the probable malingering group. This process followed the methodology described by Hubbard, Zapf, and Ronan (2003), who examined differences between defendants predicted restorable and nonrestorable to competency. All raters were blind to group membership. The data was coded categorically, and chi-square analyses and descriptive statistics were used to examine the data. The two individuals who coded the chart data included a graduate student (rater 1) completing a practicum at the psychiatric hospital who was familiar with the presentation of the information, and a psychologist at the hospital (rater 2). Both raters were trained in the format and use of the coding sheet prior to coding data and used practice charts. Rater 1 reviewed 27 charts, and Rater 2 reviewed 30 charts. This author reviewed 
approximately half of each rater's forms (total of 28) to determine inter-rater reliability. Charts were numbered without group designation (probable malingerers or honest inpatients), and a master list of group placements was used after coding procedures to run analyses.

Results

Validation of the ADI Feigning (Fg) Scale

Mean scores for the extreme groups on the ADI Depression scale score and Feigning scale score are summarized in Table 3. A one-way analysis of variance was conducted to examine group differences on the Depression scale score. A significant effect was found, $F(1$, $56)=41.01, p<.001$. The strength of this effect, as measured by eta-squared, was .43 . The honest psychiatric inpatient group $(M=36.68, S D=9.10)$ scored significantly lower on the Depression scale than did the probable malingering group $(M=53.63, S D=10.09)$. A one-way analysis of variance comparing means of the groups on the ADI Feigning scale score also revealed significant differences between groups, $F(1,56)=63.11, p<.001$. The strength of this effect, as measured by eta-squared, was .53 . The probable malingering group $(M=16.23, S D=$ 3.78) scored significantly higher on the Feigning scale score than did the honest inpatient group $(M=9.41, S D=1.71)$.

Analyses were conducted to determine the ability of the ADI Feigning scale score to detect malingering using the two extreme groups (see Table 4). The present study supported findings from previous studies with the ADI Feigning scale (Mogge, 2006), which indicated a cut-off score of 14 to be the most effective at accurate detection of group placement. Detection rates for cut-off scores of 12, 13, 15, and 16 are also included in Table 4. Sensitivity and specificity were calculated, which are, respectively, the probability that identifications of malingering are made for individuals who are malingering (sensitivity) and the likelihood that 
honest persons will be classified as honest responders (specificity). Also calculated were positive predictive power, or the percentage of individuals who were correctly identified to be malingering, and negative predictive power, or the percentage of individuals correctly identified to be honest. Hit rate calculations involved the overall percentage of individuals correctly identified between groups.

A cut-off score of 14 detected $80 \%$ ( 28 out of 35 ) of the individuals in the probable malingering group, indicating a high level of sensitivity. None of the participants in the honest inpatient group scored greater than a 14 , yielding a false positive rate of $0 \%$, or specificity of $100 \%$. Of the participants scoring below the cut-off score of 14 , only 7 were in the probable malingering group, with all 22 honest inpatients in the group as well, revealing negative predictive power (NPP) of 75.9\%. Because none of the honest inpatients scored above the cut-off score and all of those who did belonged in the probable malingering group, positive predictive power (PPP) was at $100 \%$. Among all participants, 50 of the 57 received ADI Feigning scale scores that correctly identified their group classification, yielding an overall hit rate of $87.7 \%$. Receiver Operating Characteristics revealed a ROC area under the curve at .891, or $89.1 \%$. Demographic, Clinical, and Criminal Profile Differences

Chart reviews for participants in the two most extreme groups, probable malingerers and honest psychiatric inpatients, were conducted using a standard coding sheet (Appendix A). Twenty-eight files were randomly selected (approximately half of each rater's files) to determine inter-rater reliability. As shown in Table 5, Kappa levels ranged from .69 to 1.0. Twelve of the 26 total variables (46\%) had a Kappa value of 1.00, and 20 of the 26 variables (77\%) had a Kappa value of .80 or higher. The variables with the most disagreement were Living Situation, Employment Status, Intellectual Classification, and Most Serious Type of Offense. The type of 
offense variable was often a result of failure to account for a Violent Offense charge. In all cases of disagreement, charts were reviewed by both the original rater and independent rater in an attempt to reconcile the difference and locate the correct information. Often, the information was discrepant within an individual's chart. Overall, inter-rater reliability was found to be good due to obtainment of Kappa values of .70 or higher and as compared to previous studies utilizing chart coding procedures (Hubbard, Zapf, \& Ronan, 2003).

Descriptive statistics for each group are summarized in Table 1. Within both groups, $87.7 \%$ of participants were male, and $12.3 \%$ were female. Most were Caucasian (93\%), with other races including African American (5.3\%) and Other (1.8\%). The majority of individuals were single (43.9\%), followed by those divorced (26.3\%), married (24.6\%), separated (3.5\%), and widowed (1.8\%). Additional demographic information from each of the two groups is contained in Table 1.

Using chi square analyses, demographic differences between the two groups were compared, revealing no significant differences in age, gender, race, marital status, education, living situation, employment status, and source of income. In addition, IQ scores were compared when that information was available in the charts. Nine of the individuals classified as probable malingerers had received a Wechsler Adult Intelligence Scale, $3^{\text {rd }}$ Edition Categorization $(3=$ Borderline range, $1=$ Low Average, $5=$ Average). Five of the individuals in the honest inpatient group had received a categorization $(2=$ Borderline, $1=$ Low Average, $1=$ Average, $1=$ High Average). There were no significant differences between the groups.

Chi square analyses were also used to examine clinical profile differences, which are summarized in Table 6. No significant differences were found when comparing presence of a major psychotic disorder or a major nonpsychotic disorder. However, there was a significant 
difference when comparing the presence of nonpsychotic minor disorders, which included adjustment disorders, personality disorders, and the diagnosis of malingering. $X^{2}(3, N=57)=$ $10.8, p<.05$. Of the probable malingerers, $48.6 \%$ had received a diagnosis of a nonpsychotic minor disorder that did not include malingering, and $11.4 \%$ had no diagnosis of a nonpsychotic minor disorder or malingering. Of the remaining members of this group, $9.7 \%$ had a diagnosis of malingering, and 35.5\% had a diagnosis of a minor disorder and malingering. Among those in the honest psychiatric inpatient group, 59\% had a nonpsychotic minor disorder diagnosis; however, $36 \%$ had no diagnosis in this category and only one individual had a diagnosis of a minor disorder and malingering. When personality disorders were examined separately, there were no overall group differences; however, 10 individuals in the probable malingering group had a diagnosis of Antisocial Personality Disorder compared to 2 individuals in the honest psychiatric group. Each group had two individuals diagnosed with Borderline Personality Disorder, and the honest inpatient group included one individual with a Paranoid Personality Disorder Diagnosis. Fourteen from the probable malingering group and 8 from the honest psychiatric inpatient group carried a diagnosis of Personality Disorder NOS. Nine participants from each group did not have an Axis II diagnosis.

Another statistically significant clinical difference between the groups was number of previous hospitalizations, $X^{2}(1, N=56)=7.935, p<.01$. More individuals in the probable malingering group had evidence of a previous hospitalization $(n=34)$ than in the honest psychiatric inpatient group $(n=15)$. Trends toward significant existed when considering use of psychotropic medications and previous contact with mental health services. Although 19 of the 22 participants in the honest group used psychotropic medications, all 35 of the individuals in the probable malingering group used medications. Regarding previous contact with mental health 
services, again all members of the probable malingering group had received previous mental health services, compared to 19 of those in the honest inpatient group.

Contrary to the present study's hypotheses, none of the criminal / legal history variables were significantly different between the two groups. These are summarized in Table 7 .

\section{Discussion}

As hypothesized, the Assessment of Depression Inventory (ADI; Mogge \& LePage, 2004) Depression scale scores and Feigning scale scores distinguished between the groups of participants, with probable malingerers obtaining higher scores on both scales than the honest psychiatric inpatients. Detection rates for the ADI Feigning scale were also obtained using the two extreme groups, the probable malingerers and the honest inpatient group. Using a cut-off score of 14 , the Fg scale accurately detected $80 \%$ of the probable malingerers and excluded all of the honest inpatients, yielding an overall hit rate of $87.7 \%$. This high level of sensitivity and specificity indicate that the ADI Feigning scale continues to show great promise in its ability to detect deceptive responding.

A second aim of the study involved the comparison of demographic, clinical, and criminal / legal variables between the two extreme groups in attempts to provide additional information to clinicians who utilize the threshold and clinical decision making models in the assessment of malingering (Rogers \& Bender, 2003). Similar to the purpose of studies with restorable and non-restorable defendants (Hubbard and Zapf, 2003; Hubbard, Zapf, \& Ronan, 2003), this data was not intended to be used as a sole means of determining deceptive responding, but to be included in Rogers and Bender's (2003) suggested use of multi-modal, multi-assessment strategies along with extensive review of an individual's history, the use of secondary sources of information, and the above mentioned decision making strategies. 
Interestingly, and contrary to the study's hypothesis, none of the criminal / legal variables were significantly different between the two groups, and unlike preliminary analysis of differences between malingerers and honest responders (Lewis, Simcox, \& Berry, 2002), no demographic variables were significantly different. Differences were evident within clinical variables, as evidenced by a greater number of probable malingerers receiving a nonpsychotic minor diagnosis, which included a personality disorder diagnosis (Axis II) or a diagnosis of malingering. Almost half of the probable malingerers (45\%) had received a sole diagnosis of malingering or had both a malingering and personality disorder diagnosis. Of the participants in this group, $9.7 \%$ had a diagnosis of malingering, and $35.5 \%$ had a diagnosis of a minor disorder and malingering. Among the personality disorders, it is not surprising that more individuals in the probable malingering group had been diagnosed with Antisocial Personality Disorder, as hypothesized. However, other individuals in the probable malingering group had been diagnosed with Borderline Personality Disorder, and the majority in this group had received a Personality Disorder NOS diagnosis. One possible explanation for this large number of probable malingerers with a legitimate diagnosis is that most individuals, in an inpatient setting in particular, have some type of mental health condition or disorder that may have contributed in some way to the commission of a crime or to difficulties with others in their lives. Features of several different types of personality disorders are the presence of difficult and tumultuous interpersonal relationships and problems with emotional regulation. This combination could make an individual more susceptible to assault and battery charges, domestic violence charges, or destruction of property charges. It is likely that a "pure" malingerer is rare and an exception, not the rule, to the more common presentation of malingering with a co-occurring nonpsychotic disorder. This is not to suggest that individuals who present as malingering do not or cannot have 
a co-occurring psychotic disorder or nonpsychotic major disorder. This is evident in that there was no clinically significant difference on either of these two clinical factors between the groups.

Yet another significant clinical difference between the two groups was number of previous hospitalizations, with all individuals in the probable malingering group having had a previous hospitalization. Although this finding initially seems surprising, several possible explanations could be applied. First, the above explanation for the presence of nonpsychotic minor disorders (such as personality disorders) along with malingering could apply to this finding as well. Personality disorders, particularly those with Cluster B traits, can present serious interpersonal problems for individuals, and those who are affected by them could be more likely to contact outside authorities and mental health professionals for assistance. Another explanation involves a possible tendency that for individuals who present clinically in order to receive secondary gain, they may more willingly or overtly seek inpatient treatment. Those with a legitimate serious mental illness often lack insight and fail to comply with or seek out treatment. In addition, individuals in the probable malingering group who had been hospitalized previously could have obtained knowledge about symptoms of mental illness that could assist them in feigned presentations of mental illness. These same explanations could also apply to the trends found in the present study regarding use of psychotropic medications and previous contact with mental health services. Again, all individuals in the probable malingering group used psychotropic medications and had previous contact with mental health services.

Contrary to the study's hypothesis that criminal / legal variables may be significantly different between the groups, no differences were found. Perhaps this result is due to the impact that one's psychiatric condition can have on judgment, impulse control, and ability to rationally consider the consequences of one's actions. These impairments can lead to behaviors that are 
illegal and/or harmful to others and result in legal involvement and/or a criminal charge.

When considering the findings of the present study, it appears that "red flags" typically used to alert clinicians to assess for malingering in an inpatient setting (use of the threshold model) may not be "red flags" at all. The presence of legal charges or a legal history does not necessarily indicate that one may be malingering. Similarly, having a legitimate psychiatric diagnosis, previous contact with mental health services, previous inpatient hospitalizations, and use of psychotropic medications does not indicate that one is not malingering. The importance of multi-modal, multi-assessment strategies is once again highlighted from these results (Rogers, 1997). In addition, the study's findings most likely generalize to other inpatient settings, particularly those with forensic units, and may not generalize to outpatient settings or jails / prisons.

In addition to the strong and promising findings of the present study, several limitations are necessary to mention. First, the participants received a particular battery of assessments based on treatment team decisions and individual presentation. However, it is unknown how many of those in the probable malingering group were attempting to malinger depression specifically and not psychosis or other psychiatric symptoms. As such, this validation of the ADI Feigning scale is more similar to studies examining the effectiveness of a measure's ability to detect deceptive responding in general and not depression specifically. Of note, however, is that those in the probable malingering group did obtain an overall mean score higher than the other two groups for the Depression scale, suggesting that some or all were endorsing symptoms of depression. Whether or not they were initially attempting to feign depression, it appears that their responding is likely similar to those whose intention is to malinger depression.

A further limitation to the study is the relative homogeneity of the sample and the small 
sample size. Almost all participants were Caucasian and male. Ninety percent of the participants included in the study were male, and $95.7 \%$ were Caucasian. Within the probable malingering group, only three participants were female. A more heterogeneous sample, including a larger number of females and a more diverse representation of other races, may impact the presence of demographic, clinical, and criminal / legal variable differences. It is difficult to draw general conclusions, particularly whether or not demographic differences exist between the two groups, when the sample itself is similar. A larger, more diverse sample size and replication are also needed.

Another limitation of the present study involves the difficulties inherent in coding data from inpatient charts. While Sharpe Hospital conducts periodic chart reviews for accuracy and quality assurance checks, including reviews from JCAHO, information contained in hospital charts is often incomplete or discrepant. While the current study evidenced good levels of interrater reliability, more detailed information from chart reviews would be preferred, including type of psychotropic medication and number of previous hospitalizations.

The results of this study suggest that the Assessment of Depression Inventory (ADI; Mogge \& LePage, 2004) Feigning scale is a useful assessment in detecting individuals who are attempting to feign depression or who are completing assessments with a deceptive response style. The Feigning scale demonstrated $80 \%$ sensitivity and $100 \%$ specificity using a cut score of 14 with known groups' samples. The use of multiple assessments to classify individuals as malingering is vital (Rogers, 1997), in that misclassification could prevent appropriate treatment and lead to unfair outcomes in the justice system. Failure to correctly classify individuals as malingering, however, could lead to unnecessary use of mental health care resources and injustice in the legal system. The Assessment of Depression Inventory (ADI; Mogge \& LePage, 
2004) represents an advancement in the assessment of malingering of specific disorders while also assessing for the presence of a particular disorder.

In addition, the results of this study suggest that factors previously considered to be indicative of legitimate psychiatric presentations (presence of a psychiatric diagnosis, previous hospitalizations) may not accurately represent factors that should be used to make this type of determination. Future research examining the effectiveness of the ADI Feigning scale and the clinical, demographic, and legal profile differences between probable malingerers and honest responders could include diverse samples in various forensic settings, such as outpatient clinics, private practices, and in correctional settings. 


\section{References}

American Psychiatric Association. (2000). Diagnostic and statistical manual of mental disorders ( $4^{\text {th }}$ ed., text revision). Washington, DC: Author.

Arbisi, P. A., \& Ben-Porath. (1995). An MMPI-2 infrequent response scale for use with psychopathological populations: The Infrequency-Psychopathology Scale, $F(p)$. Psychological Assessment, 7, 424-431.

Bagby, R. M., Nicholson, R. A., Bacchiochi, J. R., Ryder, A. G., \& Bury, A. S. (2002). The predictive capacity of the MMPI-2 and the PAI validity scales and indexes to detect coached and uncoached feigning. Journal of Personality Assessment, 78, 69-86.

Bagby, R. M., Marshall, M. B., \& Bacchiochi, J. R. (2005). The validity and clinical utility of the MMPI-2 Malingering Depression scale. Journal of Personality Assessment, 85, 304-311.

Beck, A. T., Steer, R. A., Brown, G. K. (1996). Manual for the Beck Depression Inventory, $2^{\text {nd }}$ ed. (BDI-II). San Antonio: The Psychological Corporation.

Bracken, B. A., \& Howell, K. (2004). Professional Manual for the Clinical Assessment of Depression (CAD). Odessa, FL: Psychological Assessment Resources.

Briere, J. (1995). Trauma Symptom Inventory professional manual. Odessa, FL: Psychological Assessment Resources.

Briere, J. (2001). DAPS: Detailed Assessment of Posttraumatic Stress professional manual. Odessa, FL: Psychological Assessment Resources.

Bury, A., S., \& Bagby, R. M. (2002). The detection of feigned uncoached and coached posttraumatic stress disorder with the MMPI-2 in a sample of workplace accident victims. Psychological Assessment, 14(4), 472-484.

Butcher, J. N., Williams, C. L., Graham, J. R., Tellegen, A., \& Kaemer, B. (1989). MMPI-2: 
Manual for administration and scoring. Minneapolis: University of Minnesota Press.

Cunnien, A. (1997). Psychiatric and medical syndromes associated with deception. In R. Rogers (Ed.), Clinical assessment of malingering and deception ( $2^{\text {nd }}$ ed., pp. 23-46). New York: Guilford Press.

Fremouw, W. J., dePerczel, M., \& Ellis, T. (1990). Suicide risk: Assessment and response guidelines. Elmsford, NY: Pergamon Press.

Fox, D. D., Gerson, A., \& Lees-Haley, P. R. (1995). Interrelationship of MMPI-2 validity scales in personal injury claims. Journal of Clinical Psychology, 51, 42-47.

Groth-Marnat, G. (2003). Handbook of psychological assessment $\left(4^{\text {th }}\right.$ ed.). Hoboken, New Jersey: John Wiley \& Sons.

Guriel, J., Yanez, R., Fremouw, W., Shreve-Neiger, A., Ware, L., Filcheck, H., \& Farr, C. (2004). Impact of coaching on malingered posttraumatic stress symptoms on the MFAST and the TSI. Journal of Forensic Psychology Practice, 4(2), 37-56.

Guy, L. S., \& Miller, H. A. (August, 2000). The M-FAST's ability to detect malingering in a prison population. Poster session presented at the $109^{\text {th }}$ annual conference of the American Psychological Association, San Francisco, CA.

Hubbard, K. L., \& Zapf, P. A. (2003). The role of demographic, criminal, and psychiatric variables in examiners' predictions of restorability to competency to stand trial. International Journal of Forensic Mental Health, 2, 145-155.

Hubbard, K. L., Zapf, P. A., \& Ronan, K. A. (2003). Competency restoration: An examination of the differences between defendants predicted restorable and not restorable to competency. Law and Human Behavior, 27(2), 127-139.

Jackson, R. L., Rogers, R., \& Sewell, K. W. (2005). Forensic applications of the Miller Forensic 
Assessment of Symptoms Test (MFAST): Screening for feigned disorders in competency to stand trial evaluations. Law and Human Behavior, 29, 199-210.

Koenig, H. G., Meador, K. G., Cohen, H. J., \& Blazer, D. G. (1988). Self-rated depression scales and screening for major depression in the older hospitalized patient with medical illness. Journal of American Geriatric Society, 36, 699-706.

Lewis, J. L, Simcox, A. M., \& Berry, D. T. R. (2002). Screening for feigned psychiatric symptoms in a forensic sample by using the MMPI-2 and the Structured Inventory of Malingered Symptomatology. Psychological Assessment, 14, 170-176.

Liljequist, L., Kinder, B. N., \& Schinka, J. A. (1998). An investigation of malingering posttraumatic stress disorder on the Personality Assessment Inventory. Journal of Personality Assessment, 71, 322-336.

Messer, J. M., \& Fremouw, W. J. (in press). Detecting malingered posttraumatic stress disorder using the Morel Emotional Numbing Test-Revised (MENT-R) and the Miller Forensic Assessment of Symptoms Test (M-FAST). Journal of Forensic Psychology Practice.

Miller, H., Guy, L. \& Davila, M. (August, 2000). Utility of the M-FAST: Detecting malingering with disability claimants. Poster session presented at the $109^{\text {th }}$ annual conference of the American Psychological Association, San Francisco, CA.

Miller, H. (2001). Manual for the Miller Forensic Assessment of Symptoms Test (M-FAST). Odessa, FL: Psychological Assessment Resources.

Mogge, N. L. (2006). The Assessment of Depression Inventory (ADI): An appraisal of validity in an inpatient sample. Depression and Anxiety, 23, 1-3.

Mogge, N. L., \& LePage, J. P. (2004). The Assessment of Depression Inventory (ADI): A new instrument used to measure depression and to detect honesty of response. Depression and 
Anxiety, 20, 107-113.

Mogge, N. L., Steinberg, J., Fremouw, W., \& Strunk, J. (in press). The Assessment of Depression Inventory (ADI): An appraisal of validity in an outpatient sample. Depression and Anxiety.

Morel, K. R. (1998). Development and preliminary validation of a forced-choice test of response bias for posttraumatic stress disorder. Journal of Personality Assessment, 70, 299-314.

Morey, L. C. (1991). Personality Assessment Inventory (PAI): Professional Manual. Odessa, FL: Psychological Assessment Resources.

Morey, L. C. (1996). An Interpretive Guide to the Personality Assessment Inventory. Odessa, FL: Psychological Assessment Resources.

Norris, M. P., \& May, M. C. (1998). Screening for malingering in a correctional setting. Law and Human Behavior, 22, 315-323.

Rehm, L. P., Mehta, P., \& Dodrill, C. L. (2001). Depression. In M. Hersen \& V. B. Van Hasselt (Eds.), Advanced abnormal psychology (2 ${ }^{\text {nd }}$ ed., pp. 307-324). New York: Kluwer Academic/Plenum Publishers.

Resnick, P. J. (1997). Malingering of posttraumatic stress disorders. In R. Rogers (Ed.), Clinical assessment of malingering and deception ( $2^{\text {nd }}$ ed.) (pp. 130-152). New York: Guilford Press.

Rogers, R. (Ed.) (1997). Clinical assessment of malingering and deception $\left(2^{\text {nd }}\right.$ ed.). New York: Guilford Press.

Rogers, R., \& Bender, S. (2003). Evaluation of malingering and deception. In A. M. Goldstein \& I. B. Weiner (Eds.), Handbook of psychology (pp. 109-129). New York: Wiley \& Sons. Rogers, R., Gillis, J. R., Bagby, R. M., \& Monteiro, E. (1991). Detection of malingering on the 
Structured Interview of Reported Symptoms (SIRS): A study of coached and uncoached simulators. Psychological Assessment: A Journal of Consulting and Clinical Psychology, 3, 673-677.

Rogers, R., Kropp, P. R., Bagby, R. M., \& Dickens, S. E. (1992). Faking specific disorders: A study of the Structured Interview of Reported Symptoms (SIRS). Journal of Clinical Psychology, 48, 643-648.

Rogers, R., Ornduff, S. R., \& Sewell, K. W. (1993). Feigning specific disorders: A study of the Personality Assessment Inventory (PAI). Journal of Personality Assessment, 60, 554560.

Rogers, R., \& Salekin, R. T. (1998). Research report beguiled by Bayes: A re-analysis of Mossman and Hart's estimates of malingering. Behavioral Sciences and the Law, 16, $147-153$.

Rogers, R., Sewell, K. W., \& Goldstein, A. (1994). Explanatory model of malingering: A prototypical analysis. Law and Human Behavior, 18, 543-552.

Rogers, R., Sewell, K. W., Morey, L. C., \& Ustad, K. L. (1996). Detection of feigned mental disorders on the Personality Assessment Inventory: A discriminant analysis. Journal of Personality Disorders, 67, 629-640.

Rogers, R., Sewell, K. W., \& Salekin, R. T. (1994). A meta-analysis of malingering on the MMPI-2. Assessment, 1, 227-237.

Scragg, P., Bor, R., \& Mendham, M. C. (2000). Feigning post-traumatic stress disorder on the PAI. Clinical Psychology and Psychotherapy, 7, 155-160.

Smith, G. P., \& Burger, G. K. (1997). Detection of malingering: Validation of the Structured Inventory of Malingered Symptomatology (SIMS). Journal of the American Academy of 
Psychiatry and Law, 25(2), 183-187.

Steffan, J. S., Clopton, J. R., \& Morgan, R. D. (2003). An MMPI-2 scale to detect malingered depression. Assessment, 10, 382-392.

Vess, J., Murphy, C., \& Arkowitz, S. (2004). Clinical and demongraphic differences between sexually violent predators and other commitment types in a state forensic hospital. The Journal of Forensic Psychiatry \& Psychology, 15, 669-681.

Walters, G. L., \& Clopton, J. R. (2000). Effect of symptom information and validity scale information on the malingering of depression on the MMPI-2. Journal of Personality Assessment, 75, 183-199.

Widows, M. R., \& Smith, G. P. (2005). Professional Manual for the Structured Inventory of Malingered Symptomatology (SIMS). Odessa, FL: Psychological Assessment Resources. 
Table 1

Comparison of Probable Malingerers and Honest Inpatients on Demographic Variables

\begin{tabular}{|c|c|c|c|c|c|}
\hline \multirow{4}{*}{ Category } & Probable & Honest & \multirow[b]{4}{*}{$X^{2}$} & \multirow[b]{4}{*}{$d f$} & \multirow[b]{4}{*}{$p$} \\
\hline & Malingerers & Inpatients & & & \\
\hline & $(\mathrm{n}=35)$ & $(\mathrm{n}=22)$ & & & \\
\hline & n $\%$ & n $\%$ & & & \\
\hline Race & & & 1.64 & 2 & .440 \\
\hline Caucasian & $33(94.3 \%)$ & $20(90.9 \%)$ & & & \\
\hline African Amer. & $2(5.7 \%)$ & $1(4.5 \%)$ & & & \\
\hline Other & 0 & $1(4.5 \%)$ & & & \\
\hline Gender & & & 1.16 & 1 & .282 \\
\hline Male & $32(91.4 \%)$ & $18(81.8 \%)$ & & & \\
\hline Female & $3(8.6 \%)$ & $4(18.2 \%)$ & & & \\
\hline Age & & & 30.98 & 27 & .272 \\
\hline 18 & $2(5.7 \%)$ & $1(4.5 \%)$ & & & \\
\hline 20 & $1(2.86 \%)$ & $1(4.5 \%)$ & & & \\
\hline 21 & $1(2.86 \%)$ & 0 & & & \\
\hline 22 & $1(2.86 \%)$ & 0 & & & \\
\hline 24 & $1(2.86 \%)$ & $1(4.5 \%)$ & & & \\
\hline 25 & $3(8.6 \%)$ & $3(13.6 \%)$ & & & \\
\hline 26 & $3(8.6 \%)$ & 0 & & & \\
\hline 27 & $2(5.7 \%)$ & $1(4.5 \%)$ & & & \\
\hline 28 & $2(5.7 \%)$ & 0 & & & \\
\hline
\end{tabular}




\begin{tabular}{|c|c|c|}
\hline 29 & 0 & $2(9.1 \%)$ \\
\hline 30 & $2(5.7 \%)$ & 0 \\
\hline 31 & $1(2.86 \%)$ & 0 \\
\hline 32 & $1(2.86 \%)$ & $2(9.1 \%)$ \\
\hline 33 & $1(2.86 \%)$ & 0 \\
\hline 34 & $1(2.86 \%)$ & 0 \\
\hline 36 & 0 & $1(4.5 \%)$ \\
\hline 37 & $2(5.7 \%)$ & $1(4.5 \%)$ \\
\hline 38 & 0 & $1(4.5 \%)$ \\
\hline 39 & $1(2.86 \%)$ & $1(4.5 \%)$ \\
\hline 42 & $1(2.86 \%)$ & 0 \\
\hline 44 & $1(2.86 \%)$ & $1(4.5 \%)$ \\
\hline 45 & 0 & $3(13.6 \%)$ \\
\hline 46 & $3(8.6 \%)$ & 0 \\
\hline 47 & 0 & $1(4.5 \%)$ \\
\hline 49 & $4(11.4 \%)$ & 0 \\
\hline 50 & 0 & $1(4.5 \%)$ \\
\hline 51 & $1(2.86 \%)$ & 0 \\
\hline 53 & 0 & $1(4.5 \%)$ \\
\hline
\end{tabular}

Marital Status

$\begin{array}{lll}2.85 & 4 & .583\end{array}$

Single

$15(42.9 \%)$

$10(45.45 \%)$

Married

$7(20 \%)$

$7(31.8 \%)$ 

Divorced
$10(28.6 \%)$
$5(22.7 \%)$

Widowed

$1(2.86 \%)$

0

Separated

$2(5.7 \%)$

0

Education

Unknown

$1(2.86 \%)$

$\begin{array}{lll}3.597 & 3 & .308\end{array}$

Elementary

$6(17.14 \%)$

$2(9.1 \%)$

High School

$21(60 \%)$

$11(50 \%)$

College

$7(20 \%)$

$9(40.9 \%)$

Living Situation

$3.123 \quad 4$

.537

Unknown

$2(5.7 \%)$

0

Alone

$3(8.6 \%)$

$2(9.1 \%)$

Homeless

$10(28.6 \%)$

$5(22.7 \%)$

Family/Friends

$20(57.1 \%)$

$14(63.6 \%)$

Employment

Unknown
Unemployed

$1(2.86 \%)$

0

Full-Time

$32(91.4 \%)$

$19(86.4 \%)$

Part-Time

$2(5.7 \%)$

$1(4.5 \%)$

0

$1(4.5 \%)$

Student

0

$1(4.5 \%)$

Source of Income

3.05

$3.884 \quad 4$

.422

Unknown

$2(5.7 \%)$

0

Unemployed

$20(57.1 \%)$

$14(63.6 \%)$

Employed

$2(5.7 \%)$

$1(4.5 \%)$ 
Disability $\quad 11(31.4 \%) \quad 6(27.3 \%)$

Family Support $\quad 0 \quad 1(4.5 \%)$

$\begin{array}{llll}\text { IQ Classification } & 2.938 & 4 & .568\end{array}$

$\begin{array}{ccc}\text { Unknown } & 26(74.3 \%) & 17(77.3 \%) \\ \text { Borderline } & 3(8.6 \%) & 2(9.1 \%) \\ \text { Low Average } & 1(2.86 \%) & 1(4.5 \%) \\ \text { Average } & 5(14.3 \%) & 1(4.5 \%)\end{array}$

High Average

$0 \quad 1(4.5 \%)$

Note. Amer. $=$ American 
Table 2

Criteria for Group Placement

\begin{tabular}{|c|c|c|c|}
\hline Group & SIRS & MMPI-2 & PAI \\
\hline & & $F>=107 T$ or $F b>=$ & NIM $T>=92$ or \\
\hline Probable & 1 scale $=$ Definite or & $108 T$ or $F-K>=$ & 3 or $>$ on MAL or \\
\hline \multirow[t]{2}{*}{ Malingerers } & 3 scales $=$ Probable & 10 or $F(p)>=100 T$ & Elevated RDF \\
\hline & SIRS scores as & & \\
\hline Indeterminate & above or invalid & & \\
\hline Malingerers & MMPI-2 or PAI & & \\
\hline Non-Malingering & No SIRS or MFAST & & \\
\hline Inpatients & $<6$ & Valid profile & Valid profile \\
\hline
\end{tabular}


Table 3

Assessment of Depression Inventory (ADI) Scale Scores Across Group Conditions

\begin{tabular}{|c|c|c|c|c|}
\hline \multirow[b]{4}{*}{ Scale } & Probable & Honest & \multirow[b]{4}{*}{$F(1,56)$} & \multirow{4}{*}{$\begin{array}{c}\text { Eta } \\
\text { squared }\end{array}$} \\
\hline & Malingerers & Inpatients & & \\
\hline & $(\mathrm{n}=35)$ & $(n=22)$ & & \\
\hline & Mean SD & Mean SD & & \\
\hline Depression & 53.63 (10.09) & $36.68(9.10)$ & $41.01 * * *$ & .427 \\
\hline Feigning & $16.23(3.78)$ & $9.41(1.71)$ & $63.11 * * *$ & .534 \\
\hline
\end{tabular}

Note. Depression $=$ Assessment of Depression Inventory Depression scale; Feigning = Assessment of Depression Inventory Feigning scale. $* * *=p<.001$. 
Table 4

Detection Rates of the ADI Feigning Scale Scores

\begin{tabular}{|c|c|c|c|c|c|}
\hline & Sensitivity & Specificity & PPP & NPP & Hit Rate \\
\hline \multicolumn{6}{|l|}{ Cutoff Score } \\
\hline 12 & $82.9 \%$ & $86.4 \%$ & $90.6 \%$ & $76 \%$ & $84.2 \%$ \\
\hline 13 & $80 \%$ & $90.9 \%$ & $93.3 \%$ & $74.1 \%$ & $84.2 \%$ \\
\hline 14 & $80 \%$ & $100 \%$ & $100 \%$ & $75.9 \%$ & $87.7 \%$ \\
\hline 15 & $71.4 \%$ & $100 \%$ & $100 \%$ & $68.8 \%$ & $82.5 \%$ \\
\hline 16 & $51.4 \%$ & $100 \%$ & $100 \%$ & $56.4 \%$ & $70.2 \%$ \\
\hline
\end{tabular}

Note. PPP = Positive Predictive Power; NPP = Negative Predictive Power; ROC = Receiver Operating Characteristics 
Table 5

Inter-rater Reliability for Demographic, Clinical, and Criminal / Legal Variables

Variable

Cohen's Kappa

Age

Gender

0.87

Ethnicity

1.00

Marital Status

0.95

Education Level

Living Situation

Employment Status

0.73

Source of Income

Intellectual Classification

0.74

Diagnosed with Psychotic disorder

1.00

Diagnosed with Non-psychotic Major Disorder

Diagnosed with Non-psychotic Minor Disorder

Type of Personality Disorder

Diagnosed with Alcohol Use Disorder

Diagnosed with Drug Use Disorder

1.00

Diagnosed with Alcohol and Drug Use Disorder

Most Serious Type of Disorder

0.95

Previous Mental Health Services

1.00

Previous Hospitalizations

Use of Psychotropic Medications

0.94 


\begin{tabular}{lc}
\hline Charged with Violent Offense & 0.79 \\
Charged with Property Offense & 0.94 \\
Charged with Miscellaneous Offense & 0.85 \\
Most Serious Type of Offense & 0.74 \\
Charged with Murder & 1.00 \\
Previous Criminal History & 0.75 \\
\hline
\end{tabular}


Table 6

Comparison of Probable Malingerers and Honest Inpatients on Clinical Variables

\begin{tabular}{|c|c|c|c|c|c|}
\hline \multirow{4}{*}{ Category } & Probable & Honest & \multirow[b]{4}{*}{$X^{2}$} & \multirow[b]{4}{*}{$d f$} & \multirow[b]{4}{*}{$p$} \\
\hline & Malingerers & Inpatients & & & \\
\hline & $(\mathrm{n}=35)$ & $(n=22)$ & & & \\
\hline & n $\%$ & $\mathrm{n} \%$ & & & \\
\hline Psychotic Disorder & & & .380 & 1 & .538 \\
\hline Yes & $10(28.6 \%)$ & $8(36.4 \%)$ & & & \\
\hline No & $25(71.4 \%)$ & $14(63.6 \%)$ & & & \\
\hline \multicolumn{6}{|l|}{ Nonpsychotic Major } \\
\hline Disorder & & & .011 & 1 & .915 \\
\hline Yes & $10(28.6 \%)$ & $6(27.3 \%)$ & & & \\
\hline No & $25(71.4 \%)$ & $16(72.7 \%)$ & & & \\
\hline \multicolumn{6}{|l|}{ Nonpsychotic Minor } \\
\hline Disorder & & & 10.797 & 3 & $.013 *$ \\
\hline Yes & $17(48.6 \%)$ & $13(59 \%)$ & & & \\
\hline No & $4(11.4 \%)$ & $8(36.4 \%)$ & & & \\
\hline Malingering & $3(8.6 \%)$ & 0 & & & \\
\hline \multicolumn{6}{|l|}{ Malingering and } \\
\hline Minor Disorder & $11(31.4 \%)$ & $1(4.5 \%)$ & & & \\
\hline Personality Disorder & & & 5.28 & 4 & .260 \\
\hline None & $9(25.7 \%)$ & $9(40.9 \%)$ & & & \\
\hline Paranoid & 0 & $1(4.5 \%)$ & & & \\
\hline
\end{tabular}




\begin{tabular}{|c|c|c|c|c|c|}
\hline Antisocial & $10(28.6 \%)$ & $2(9.1 \%)$ & & & \\
\hline Borderline & $2(5.7 \%)$ & $2(9.1 \%)$ & & & \\
\hline NOS & $14(40 \%)$ & $8(36.4 \%)$ & & & \\
\hline Alcohol Use D/O & & & .338 & 1 & .561 \\
\hline Yes & $5(14.3 \%)$ & $2(9.1 \%)$ & & & \\
\hline No & $30(85.7 \%)$ & $20(90.9 \%)$ & & & \\
\hline Drug Use D/O & & & .368 & 1 & .544 \\
\hline Yes & $3(8.6 \%)$ & $3(13.6 \%)$ & & & \\
\hline No & $32(91.4 \%)$ & $19(86.4 \%)$ & & & \\
\hline Alcohol and Drug & & & 2.11 & 1 & .146 \\
\hline Yes & $18(51.4 \%)$ & $7(31.8 \%)$ & & & \\
\hline No & $17(48.6 \%)$ & $15(68.2 \%)$ & & & \\
\hline Most Serious D/O & & & .695 & 3 & .874 \\
\hline Psychotic & $10(28.6 \%)$ & $8(36.4 \%)$ & & & \\
\hline Nonpsychotic Major & $7(20 \%)$ & $5(22.7 \%)$ & & & \\
\hline Nonpsychotic Minor & $7(20 \%)$ & $4(18.2 \%)$ & & & \\
\hline Drug or Alcohol & $11(31.4 \%)$ & $5(22.7 \%)$ & & & \\
\hline \multicolumn{6}{|l|}{ Previous } \\
\hline Hospitalization & & & 7.935 & 1 & $.005^{* *}$ \\
\hline Yes & $34(97.1 \%)$ & $15(68.2 \%)$ & & & \\
\hline No & $1(2.86 \%)$ & $6(27.3 \%)$ & & & \\
\hline \multicolumn{6}{|l|}{ Psychotropic } \\
\hline Medication & & & 3.457 & 1 & .063 \\
\hline
\end{tabular}




$\begin{array}{ccc}\text { Yes } & 35(100 \%) & 19(86.4 \%) \\ \text { No } & 0 & 2(9 \%)\end{array}$

\section{Previous Contact}

Mental Health

$\begin{array}{lll}3.457 & 1 & .063\end{array}$

Yes

$35(100 \%)$

$19(86.4 \%)$

No

0

$2(9 \%)$

Note. $\mathrm{D} / \mathrm{O}=$ Disorder; NOS $=$ Not Otherwise Specified; NOS = Not Otherwise Specified; $*=p<.05 ; * *=p<.01$ 
Table 7

Comparison of Probable Malingerers and Honest Inpatients on Criminal/Legal Variables

\begin{tabular}{|c|c|c|c|c|c|}
\hline \multirow{4}{*}{ Category } & Probable & Honest & \multirow[b]{4}{*}{$X^{2}$} & \multirow[b]{4}{*}{$d f$} & \multirow[b]{4}{*}{$p$} \\
\hline & Malingerers & Inpatients & & & \\
\hline & $(n=35)$ & $(n=22)$ & & & \\
\hline & $\mathrm{n} \%$ & n $\%$ & & & \\
\hline Violent Offense & & & .485 & 1 & .486 \\
\hline Yes & $19(54.3 \%)$ & $14(63.6 \%)$ & & & \\
\hline No & $16(45.7 \%)$ & $8(36.4 \%)$ & & & \\
\hline Property Offense & & & .307 & 1 & .579 \\
\hline Yes & $12(34.3 \%)$ & $6(27.3 \%)$ & & & \\
\hline No & $23(65.7 \%)$ & $16(72.7 \%)$ & & & \\
\hline \multicolumn{6}{|l|}{ Miscellaneous } \\
\hline Offense & & & 1.236 & 1 & .266 \\
\hline Yes & $17(48.6 \%)$ & $14(63.6 \%)$ & & & \\
\hline No & $18(51.4 \%)$ & $8(36.4 \%)$ & & & \\
\hline \multicolumn{6}{|l|}{ Most Serious } \\
\hline Offense & & & 3.73 & 3 & .292 \\
\hline Violent & $19(54.3 \%)$ & $14(63.6 \%)$ & & & \\
\hline Property & $4(11.4 \%)$ & $2(9.1 \%)$ & & & \\
\hline Miscellaneous & $5(14.3 \%)$ & 0 & & & \\
\hline None & $7(20 \%)$ & $6(27.3 \%)$ & & & \\
\hline Murder Charge & & & .236 & 1 & .627 \\
\hline
\end{tabular}




$\begin{array}{lll}\text { Yes } & 2(5.7 \%) & 2(9.1 \%) \\ \text { No } & 33(94.3 \%) & 20(90.9 \%)\end{array}$

Criminal History

$\begin{array}{lll}.011 & 1 & .915\end{array}$

Yes

$25(71.4 \%) \quad 16(72.7 \%)$

No $\quad 10(28.6 \%)$

$6(27.3 \%)$

Note. Type of offense was based on having a legal charge. 
Appendix A

CODING FORM: CLINICAL / DEMOGRAPHIC PROFILE DIFFERENCES

\section{DEMOGRAPHIC INFORMATION}

1. Age

2. Gender

$\begin{array}{ll}\text { Male } & 1 \\ \text { Female } & 2\end{array}$

3. Ethnicity

$\begin{array}{ll}\text { Caucasian } & 1 \\ \text { African American } & 2 \\ \text { Hispanic } & 3 \\ \text { Native American } & 4 \\ \text { Asian } & 5 \\ \text { Bi-Racial } & 6 \\ \text { Other } & 7\end{array}$

4. Marital Status

Single $\quad 1$

Married 2

Divorced 3

Separated 4

Widowed 5

5. Education Level

$\begin{array}{ll}\text { Unknown } & 1 \\ \text { Elementary } & 2 \\ \text { High School } & 3 \\ \text { College } & 4 \\ \text { Vocational } & 5\end{array}$

6. Living Situation (outside of hospital; prior to admission)

$\begin{array}{ll}\text { Unknown } & 1 \\ \text { Alone Independently } & 2 \\ \text { Homeless } & 3 \\ \text { Family/Friends } & 4\end{array}$

7. Employment Status (prior to admission)

Unknown 1

Unemployed 2

Employed Full Time 3

Employed Part Time 4 
Student 5

Other 6

8. Source of Income (prior to admission)

Unknown 1

Unemployed 2

Employed FT or PT 3

Disability 4

Family Support 5

9. Intellectual Classification (According to WAIS-III Categorization)

Extremely Low (69 and below) 1

Borderline (70-79) 2

Low Average (80-89) 3

Average (90-109) 4

High Average (110-119) 5

Superior (120-129) 6

Very Superior (130 and above) 7

\section{PSYCHIATRIC VARIABLES}

*Based on most recent (current) diagnosis from unit psychiatrist

1. Diagnosed with psychotic disorder (schizophrenia; psychosis NOS; mood disorder w/ psychotic features)

YES (1)

NO (2)

2. Diagnosed with nonpsychotic major disorder (major depression; bipolar disorder; organicity)

YES (1)

NO (2)

3. Diagnosed with nonpsychotic minor disorders (adjustment disorders; personality disorders)

YES (1)

NO (2)

Malingering (3)

Malingering and other minor (4)

4. Type of personality disorder

Paranoid Personality Disorder 1

Schizoid Personality Disorder 2

Schizotypal Personality Disorder 3

Antisocial Personality Disorder 4

Borderline Personality Disorder 5

Histrionic Personality Disorder 6

Narcissistic Personality Disorder 7 
Avoidant Personality Disorder 8

Dependent Personality Disorder 9

Obsessive-Compulsive Personality Disorder 10

Personality Disorder NOS

5. Alcohol use disorder

YES (1)

NO (2)

6. Drug use disorder

YES (1)

NO (2)

7. Alcohol and drug use disorder (Both)

YES (1)

NO (2)

8. Most serious type of disorder

Psychotic 1

Nonpsychotic major disorder 2

Nonpsychotic minor disorder 3

Drug or alcohol use disorder 4

No diagnosis 5

9. Previous contact with mental health services

YES (1)

NO (2)

10. Previous hospitalizations

YES (1)

NO (2)

11. Psychotropic medications

YES (1)

NO (2)

\section{CRIMINAL / LEGAL HISTORY}

1. Charged with a violent offense (against another person)

YES (1)

NO (2)

2. Charged with a property offense

YES (1)

NO (2) 
3. Charged with a miscellaneous offense

YES (1)

NO (2)

4. Most serious type of offense

Violent 1

Property 2

Miscellaneous 3

5. Charged with murder

YES (1)

NO (2)

6. Presence of previous criminal history

YES (1)

NO (2) 